\title{
Higgs characterisation via vector-boson fusion and associated production: NLO and parton-shower effects
}

\author{
Fabio Maltoni ${ }^{1}$, Kentarou Mawatari ${ }^{2, a}$, Marco Zaro ${ }^{3}$ \\ ${ }^{1}$ Centre for Cosmology, Particle Physics and Phenomenology (CP3), Université Catholique de Louvain, 1348 Louvain-la-Neuve, Belgium \\ 2 Theoretische Natuurkunde and IIHE/ELEM, Vrije Universiteit Brussel, and International Solvay Institutes, Pleinlaan 2, 1050 Brussels, Belgium \\ ${ }^{3}$ LPTHE, CNRS UMR 7589, UPMC Univ. Paris 6, 75252 Paris, France
}

Received: 18 November 2013 / Accepted: 5 December 2013 / Published online: 25 January 2014

(C) The Author(s) 2014. This article is published with open access at Springerlink.com

\begin{abstract}
Vector-boson fusion and associated production at the LHC can provide key information on the strength and structure of the Higgs couplings to the Standard Model particles. Using an effective field theory approach, we study the effects of next-to-leading order (NLO) QCD corrections matched to a parton shower on selected observables for various spin-0 hypotheses. We find that inclusion of NLO corrections is needed to reduce the theoretical uncertainties on the total rates as well as to reliably predict the shapes of the distributions. Our results are obtained in a fully automatic way via FEYNRULES and MADGRAPH5_AMC@NLO.
\end{abstract}

\section{Introduction}

After the discovery of a new boson at the LHC [1,2], studies of its properties have become the first priority of the highenergy physics community. A coordinated theoretical and experimental effort is made [3-5] that aims at maximising the information from the ongoing and forthcoming measurements. On the experimental side, new analyses, strategies and more precise measurements are being performed that cover the wider range of relevant production and decay channels in the Standard Model (SM) and beyond, and the recent measurements of the coupling strength $[6,7]$ and the spin-parity properties $[8,9]$ give strong indications that the new particle is indeed the scalar boson predicted by the SM. On the theoretical side, predictions for signal and background are being obtained at higher orders in perturbative expansion in QCD and electroweak (EW) theory, so that a better accuracy in the extraction of the SM parameters can be achieved. In addition, new variables and observables are being proposed that may be sensitive to new physics effects. At the same time, considerable attention is being devoted to the defini-

\footnotetext{
a e-mail: kentarou.mawatari@vub.ac.be
}

tion of a theoretical methodology and framework to collect and interpret the constraints coming from the experimental side.

The proposal of employing an effective field theory (EFT) that features only SM particles and symmetries at the EW scale has turned out to be particularly appealing. Such a minimal assumption, certainly well justified by the present data, provides not only a drastic reduction of all possible interactions that Lorentz symmetry alone would allow, but also a well-defined and powerful framework where constraints coming from Higgs measurements can be globally analysed together with those coming from precision EW measurements and flavour physics (see for example Refs. [10-34], and more in general Refs. [35,36]).

In this context, the Higgs Characterisation (HC) framework has recently been presented [37], which follows the general strategy outlined in Ref. [38]. A simple EFT lagrangian featuring bosons with various spin-parity assignments has been implemented in FeYNRULES $[39,40]$ and passed to the MADGRAPH5_AMC@NLO [41-43] framework by means of the UFO model file $[44,45]$. Such an implementation is simple but general enough to describe any new physics effects coming from higher scales in a fully modelindependent way. It has the advantage of being systematically and seamlessly improvable through the inclusion of more operators in the lagrangian on one side and of higherorder corrections, notably those coming from QCD, on the other. The latter, considered in the form of multi-parton treelevel computations (ME+PS) and of next-to-leading order (NLO) calculations matched to parton showers (NLO+PS), are a very important ingredient for performing sensible phenomenological studies.

In Ref.[37] we have provided a study of higher-order QCD effects for inclusive $p p \rightarrow X\left(J^{P}\right)$ production, with $J^{P}=0^{+}, 0^{-}, 1^{+}, 1^{-}$, and $2^{+}$, and correlated decay of resonances into a pair of gauge bosons, where gluon fusion 
( $q \bar{q}$ annihilation) is dominant for spin-0 and spin-2 (spin1) at the LO. In this work, we present the results for the next most important production channels at the LHC, i.e., weak vector-boson fusion (VBF) and associated production $(\mathrm{VH})$, focusing on the most likely spin-0 hypothesis. As already noted in Ref. [37], these processes share the property that NLO QCD corrections factorise exactly with respect to the new physics interactions in Higgs couplings and therefore can be automatically performed within the current MADGRAPH5_AMC@NLO framework. Given that the Higgs characterisation can also be done automatically in the $t \bar{t} H$ production channel [46], all the main Higgs production channels are covered.

We stress that the spin-parity studies in VBF and VH production nicely complement those in $H \rightarrow Z Z / W W$ decays $[47,48]$. One of the advantages in the VBF and VH channels is that spin-parity observables, e.g., the azimuthal difference between the two tagging jets $\Delta \phi_{j j}$ in $\mathrm{VBF}$, do not require a reconstruction of the Higgs resonance, although the separation between the $Z$ and $W$ contributions is very difficult. In this study, we focus on the effects of the QCD corrections in Higgs $\mathrm{VBF}$ and $\mathrm{VH}$ production without considering the decay.

The paper is organised as follows. In the following section we recall the relevant effective lagrangian of Ref. [37], and we define the sample scenarios used to illustrate the phenomenological implications. In Sect. 3 we present the VBF results in the form of distributions of key observables in the inclusive setup as well as with dedicated VBF cuts, while in Sect. 4 we illustrate the $W^{ \pm} H$ and $Z H$ production. We briefly summarise our findings in the concluding section.

\section{Theoretical setup}

In this section, we summarise the full setup, from the lagrangian, to the choice of benchmark scenarios, to event generation at NLO accuracy.

\subsection{Effective lagrangian and benchmark scenarios}

We construct an effective lagrangian below the electroweak symmetry breaking (EWSB) scale in terms of mass eigenstates. Our assumptions are simply that the resonance structure observed in data corresponds to one bosonic state $\left(X\left(J^{P}\right)\right.$ with $J=0,1$ or 2 and a mass of about $\left.125 \mathrm{GeV}\right)$, and that no other new state below the cutoff $\Lambda$ coupled to such a resonance exists. We also follow the principle that any new physics is dominantly described by the lowest-dimensional operators. This means, for the spin-0 case, that we include all effects coming from the complete set of dimension-six operators with respect to the SM gauge symmetry.
The effective lagrangian relevant for this work, i.e., for the interactions between a spin-0 state and vector bosons, is (Eq. (2.4) in Ref. [37]):

$$
\begin{aligned}
\mathcal{L}_{0}^{V}= & \left\{c_{\alpha} \kappa_{\mathrm{SM}}\left[\frac{1}{2} g_{H Z Z} Z_{\mu} Z^{\mu}+g_{H W W} W_{\mu}^{+} W^{-\mu}\right]\right. \\
& -\frac{1}{4}\left[c_{\alpha} \kappa_{H \gamma \gamma} g_{H \gamma \gamma} A_{\mu \nu} A^{\mu \nu}+s_{\alpha} \kappa_{A \gamma \gamma} g_{A \gamma \gamma} A_{\mu \nu} \widetilde{A}^{\mu \nu}\right] \\
& -\frac{1}{2}\left[c_{\alpha} \kappa_{H Z \gamma} g_{H Z \gamma} Z_{\mu \nu} A^{\mu \nu}+s_{\alpha} \kappa_{A Z \gamma} g_{A Z \gamma} Z_{\mu \nu} \widetilde{A}^{\mu \nu}\right] \\
& -\frac{1}{4}\left[c_{\alpha} \kappa_{H g g} g_{H g g} G_{\mu \nu}^{a} G^{a, \mu \nu}+s_{\alpha} \kappa_{A g g} g_{A g g} G_{\mu \nu}^{a} \widetilde{G}^{a, \mu \nu}\right] \\
& -\frac{1}{4} \frac{1}{\Lambda}\left[c_{\alpha} \kappa_{H Z Z} Z_{\mu \nu} Z^{\mu \nu}+s_{\alpha} \kappa_{A Z Z} Z_{\mu \nu} \widetilde{Z}^{\mu \nu}\right] \\
& -\frac{1}{2} \frac{1}{\Lambda}\left[c_{\alpha} \kappa_{H W W} W_{\mu \nu}^{+} W^{-\mu \nu}+s_{\alpha} \kappa_{A W W} W_{\mu \nu}^{+} \widetilde{W}^{-\mu \nu}\right] \\
& -\frac{1}{\Lambda} c_{\alpha}\left[\kappa_{H \partial \gamma} Z_{\nu} \partial_{\mu} A^{\mu \nu} \kappa_{H \partial Z} Z_{\nu} \partial_{\mu} Z^{\mu \nu}\right. \\
& \left.\left.+\left(\kappa_{H \partial W} W_{\nu}^{+} \partial_{\mu} W^{-\mu \nu}+h . c .\right)\right]\right\} X_{0},
\end{aligned}
$$

where the (reduced) field strength tensors are defined as

$$
\begin{aligned}
& V_{\mu \nu}=\partial_{\mu} V_{\nu}-\partial_{\nu} V_{\mu} \quad\left(V=A, Z, W^{ \pm}\right), \\
& G_{\mu \nu}^{a}=\partial_{\mu} G_{\nu}^{a}-\partial_{\nu} G_{\mu}^{a}+g_{s} f^{a b c} G_{\mu}^{b} G_{\nu}^{c},
\end{aligned}
$$

and the dual tensor is

$\widetilde{V}_{\mu \nu}=\frac{1}{2} \epsilon_{\mu \nu \rho \sigma} V^{\rho \sigma}$

Our parametrisation: (i) allows one to recover the SM case easily by the dimensionless coupling parameters $\kappa_{i}$ and the dimensionful couplings $g_{X y y^{\prime}}$ shown in Tables 1 and 2; (ii) includes $0^{-}$state couplings typical of SUSY or of generic two-Higgs-doublet models (2HDM); (iii) describes $\mathrm{CP}$-mixing between $0^{+}$and $0^{-}$states, parametrised by an angle $\alpha$, in practice $-1<c_{\alpha}(\equiv \cos \alpha)<1$.

Table 1 HC model parameters

\begin{tabular}{ll}
\hline Parameter & Description \\
\hline$\Lambda(\mathrm{GeV})$ & Cutoff scale \\
$c_{\alpha}(\equiv \cos \alpha)$ & Mixing between $0^{+}$and $0^{-}$ \\
$\kappa_{i}$ & Dimensionless coupling parameter \\
\hline
\end{tabular}

Table 2 Values in units of $v$ taken by the couplings $g_{X y y^{\prime}}$ for the EW gauge bosons. $C=\sqrt{\frac{\alpha_{\mathrm{EM}} G_{F} m_{Z}^{2}}{8 \sqrt{2} \pi}}$

\begin{tabular}{llll}
\hline$g_{X y y^{\prime}} \times v$ & $Z Z / W W$ & $\gamma \gamma$ & $Z \gamma$ \\
\hline$X=H$ & $2 m_{Z / W}^{2}$ & $47 \alpha_{\mathrm{EM}} / 18 \pi$ & $C\left(94 c_{W}^{2}-13\right) / 9 \pi$ \\
$X=A$ & 0 & $4 \alpha_{\mathrm{EM}} / 3 \pi$ & $2 C\left(8 c_{W}^{2}-5\right) / 3 \pi$ \\
\hline
\end{tabular}


Table 3 Benchmark scenarios

\begin{tabular}{ll}
\hline Scenario & HC parameter choice \\
\hline $0^{+}(\mathrm{SM})$ & $\kappa_{\mathrm{SM}}=1\left(c_{\alpha}=1\right)$ \\
$0^{+}(\mathrm{HD})$ & $\kappa_{H Z Z, H W W}=1\left(c_{\alpha}=1\right)$ \\
$0^{+}(\mathrm{HDder})$ & $\kappa_{H \partial Z, H \partial W}=1\left(c_{\alpha}=1\right)$ \\
$0^{+}(\mathrm{SM}+\mathrm{HD})$ & $\kappa_{S M, H Z Z, H W W}=1\left(c_{\alpha}=1, \Lambda=v\right)$ \\
$0^{-}(\mathrm{HD})$ & $\kappa_{A Z Z, A W W}=1\left(c_{\alpha}=0\right)$ \\
$0^{ \pm}(\mathrm{HD})$ & $\kappa_{H Z Z, A Z Z, H W W, A W W}=1\left(c_{\alpha}=1 / \sqrt{2}\right)$ \\
\hline
\end{tabular}

The corresponding implementation of the dimension-six lagrangian above the EWSB scale, where $S U(2)_{L} \times U(1)_{Y}$ is an exact symmetry, has recently appeared [49], which has overlapping as well as complementary features with respect to our HC lagrangian. We note that the lagrangian of Eq. (1) features 14 free parameters, of which one possibly complex $\left(\kappa_{\text {HəW }}\right)$. On the other hand, as explicitly shown in Table 1 of Ref. [49] these correspond to 11 free parameters in the parametrisation above the EWSB due to the custodial symmetry. We stress that the results at NLO in QCD accuracy shown here can be obtained for that lagrangian in exactly the same way.

In Table 3 we list the representative scenarios that we later use for illustration. The first corresponds to the SM. The second scenario, $0^{+}(\mathrm{HD})$, includes only the CP-even higher-dimensional operators corresponding to $\kappa_{H Z Z, H W W}$ in a custodial invariant way for VBF. The third scenario, $0^{+}$ (HDder), includes the so-called derivative operators which, via the equations of motions, can be linked to contact operators of the type $H V f f^{\prime}$. The fourth scenario, $0^{+}(\mathrm{SM}+\mathrm{HD})$, features the interference, which scales as $1 / \Lambda$ in the physical observables, between the SM and the HD operators. The fifth scenario, $0^{-}(\mathrm{HD})$, is the analogous of the second one, but for a pseudoscalar. Finally, the sixth scenario, $0^{ \pm}(\mathrm{HD})$, is representative of a CP-mixed case, where the scalar is a scalar/pseudoscalar state in equal proportion.

\subsection{NLO corrections including parton-shower effects}

The MADGRAPH5_AMC@NLO framework is designed to automatically perform the computation of tree-level and NLO cross sections, possibly including their matching to parton showers and the merging of samples with different parton multiplicities. Currently, the full automation is available in a unique and self-contained framework based on MADGRAPH5 [41] for SM processes with NLO QCD corrections. User intervention is limited to the input of physical quantities, and after event generation, to the choice of observables to be analysed. In Ref. [37] the results for gluon fusion have been presented and compared to predictions coming from ME+PS (MLM- $k_{T}$ merging [50-52]) and NLO +PS. The distributions were found to be compatible between the two predictions. In this work we limit ourselves to NLO+PS results as typical observables are inclusive in terms of extra radiation and such calculations do also provide a reliable normalisation.

AMC@NLO implements matching of any NLO QCD computation with parton showers following the MC@NLO approach [53]. Two independent and modular parts are devoted to the computation of specific contributions to an NLO-matched computation: MADFKS [42] takes care of the Born, the real-emission amplitudes, and it also performs the subtraction of the infrared singularities and the generation of the Monte Carlo subtraction terms, according to the FKS prescription [54,55]. MADLOOP [43] computes the one-loop amplitudes, using the CutTools [56] implementation of the OPP integrand-reduction method [57]. The OPENLOOPS method [58] is also used for better performance. Once the process of interest is specified by the user, the generation of the code is fully automated. Basic information, however, must be available as regards the model and the interactions of its particles with QCD partons. For MADFKS this amounts to the ordinary Feynman rules. For MADLOOP, on the other hand, the Feynman rules, UV counterterms, and special tree-level rules, so-called $R_{2}$, necessary to (and defined by) the OPP method, should be provided. While Feynman rules are automatically computed from a given lagrangian (via FEYNRULES $[39,40]$ ), this is not yet possible for $\mathrm{UV}$ counterterms and $R_{2}$ rules. At this moment this limitation hampers the automatic computation of NLO QCD corrections for arbitrary processes in generic BSM models, including the HC model. The processes considered in this paper, VBF and $\mathrm{VH}$, are, however, a notable exception as QCD corrections can be computed automatically and in full generality. This is because the corresponding one-loop amplitudes only include SM particles and do not need any UV counterterms and $R_{2}$ information from the $\mathrm{HC}$ lagrangian. In the case of VBF, this assumes that only vertex loop corrections can be computed, i.e., the pentagon diagrams are discarded, as the contributions only affect interferences between the diagrams, which are negligible already at LO.

\subsection{Simulation parameters}

In our simulations we generate events at the LHC with a centre-of-mass energy $\sqrt{s}=8 \mathrm{TeV}$ and set the resonance mass to $m_{X_{0}}=125 \mathrm{GeV}$. Parton distribution functions (PDFs) are evaluated by using the MSTW2008 (LO/NLO) parametrisation [59], and jets are reconstructed via the anti$k_{T}(\Delta R=0.4)$ algorithm [60] as implemented in FASTJET [61]. Central values for the renormalisation and factorisation scales $\mu_{R, F}$ are set to $\mu_{0}=m_{W}$ and $m_{\mathrm{VH}}$ for $\mathrm{VBF}$ and $\mathrm{VH}$ production, respectively, where $m_{\mathrm{VH}}$ is the invariant mass of the VH system. We note here that scale (and PDF) uncertainties can be evaluated automatically in the code via a reweight- 
ing technique [62], the user only deciding the range of the variation. In addition, such information is available on an event-by-event basis and therefore uncertainty bands can be plotted for any observable of interest. In this work, however, to simplify the presentation that focuses on the differences between the various scenarios, we give this information only for total cross sections and refrain from showing them in the differential distributions. For parton shower and hadronisation we employ HERWIG6 [63] in this paper, while HERWIG++ [64], (virtuality ordered) PYTHIA6 [65] and PYTHIA8 [66] are available for use in AMC@NLO. The comparison among the above different shower schemes was done for the SM Higgs boson in VBF in Ref. [67].

\section{Vector-boson fusion}

Predictions for Higgs production via VBF in the SM are known up to NNLO accuracy for the total cross section [6870], at the NLO QCD [71-76] + EW [77,78] level in a differential way and at NLO in QCD plus parton shower both in the POWHEG BOX [79] and in AMC@NLO [67]. NLO QCD predictions that include anomalous couplings between the Higgs and a pair of vector bosons are available in VBFNLO $[80,81]$. Our implementation provides the first predictions for EFT interactions including NLO corrections in QCD interfaced with a parton shower. Many phenomenological studies on Higgs spin, parity and couplings are available in the literature $[47,48,82-88]$, which could now be upgraded to NLO+PS accuracy.

In our framework the code and events for VBF can be automatically generated by issuing the following commands (note the $\$ \$$ sign to forbid diagrams with $W^{ \pm}$or $Z$ bosons in the $s$-channel which are included in $\mathrm{VH}$ production):

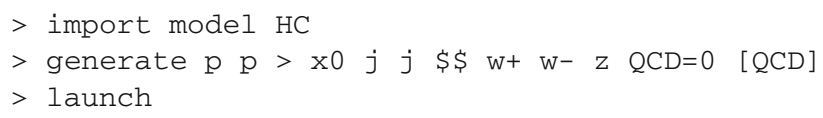

As a result all processes featuring a $V V^{\prime} \rightarrow X_{0}$ vertex, with $V=W, Z, \gamma$, are generated, therefore including $\gamma \gamma \rightarrow X_{0}$ and $Z \gamma \rightarrow X_{0}$. We do not investigate their effects in our illustrative studies below (i.e., we set the corresponding $\kappa_{i}$ to zero in the simulation), as we focus on SM-like VBF observables. As mentioned above, since our interest is geared towards QCD effects on production distributions, we do not include Higgs decays in our studies either. We stress, however, that decays (as predicted in the HC model) can be efficiently included at the partonic event level (before passing the event to a shower program) via MADSPIN [89].

In Table 4, we first collect results for total cross sections at LO and NLO accuracy together with scale uncertainties and corresponding $K$-factors for the six scenarios defined in Table 3. We do not impose any cuts here, and hence the cross sections are identical with and without par-
Table 4 VBF total cross sections with scale uncertainties and corresponding $K$-factors at $\mathrm{LHC} 8 \mathrm{TeV}$ for various scenarios

\begin{tabular}{llll}
\hline Scenario & $\sigma_{\mathrm{LO}}(\mathrm{fb})$ & $\sigma_{\mathrm{NLO}}(\mathrm{fb})$ & $K$ \\
\hline $0^{+}(\mathrm{SM})$ & $1509(1)_{-4.4 \%}^{+4.7 \%}$ & $1633(2)_{-1.5 \%}^{+2.0 \%}$ & 1.08 \\
$0^{+}(\mathrm{HD})$ & $69.66(6)_{-6.6 \%}^{+7.5 \%}$ & $67.08(13)_{-2.3 \%}^{+2.2 \%}$ & 0.96 \\
$0^{+}(\mathrm{HDder})$ & $721.9(6)_{-9.0 \%}^{+11.0 \%}$ & $684.9(1.5)_{-2.8 \%}^{+2.3 \%}$ & 0.95 \\
$0^{+}(\mathrm{SM}+\mathrm{HD})$ & $3065(2)_{-5.1 \%}^{+5.6 \%}$ & $3144(5)_{-1.1 \%}^{+1.6 \%}$ & 1.03 \\
$0^{-}(\mathrm{HD})$ & $57.10(4)_{-6.7 \%}^{+7.7 \%}$ & $55.24(11)_{-2.5 \%}^{+2.1 \%}$ & 0.97 \\
$0^{ \pm}(\mathrm{HD})$ & $63.46(5)_{-6.7 \%}^{+7.6 \%}$ & $61.07(13)_{-2.0}^{+2.3 \%}$ & 0.96 \\
\hline
\end{tabular}

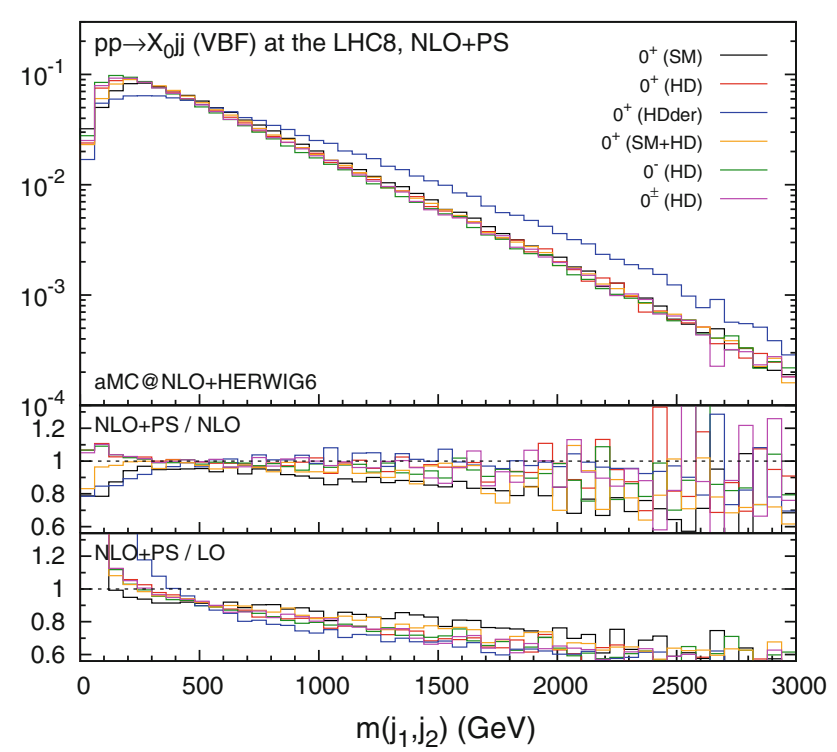

Fig. 1 Distribution for the invariant mass of the two leading jets in VBF production with the acceptance cuts. The histograms in the main plot are normalised to unity

ton shower. The cross sections for the HD hypotheses are calculated with the corresponding $\kappa_{i}$ set to 1 and the cutoff scale $\Lambda=1 \mathrm{TeV}$ except for the $0^{+}(\mathrm{SM}+\mathrm{HD})$ scenario, where we set $\Lambda=v=246 \mathrm{GeV}$. We do this to allow for visible effects of the interference between the SM and HD terms. Equivalently, we could have kept $\Lambda=1 \mathrm{TeV}$ and chosen a larger value for $\kappa_{i}$, as only the ratio $\kappa_{i} / \Lambda$ is physical. The figures in parentheses give the numerical integration uncertainties in the last digit(s). The other uncertainties correspond to the envelope obtained by varying independently the renormalisation and factorisation scales around the central value $1 / 2<\mu_{R, F} / \mu_{0}<2$ with $\mu_{0}=m_{W}$. NLO QCD corrections contribute constructively for the SM case, but destructively for the HD cases, although the global $K$-factors are rather mild. The uncertainties in the HD scenarios, especially for the derivative operator (HDder), are larger than that in the SM case. Manifestly, the uncertainties are significantly reduced going from LO to NLO. 

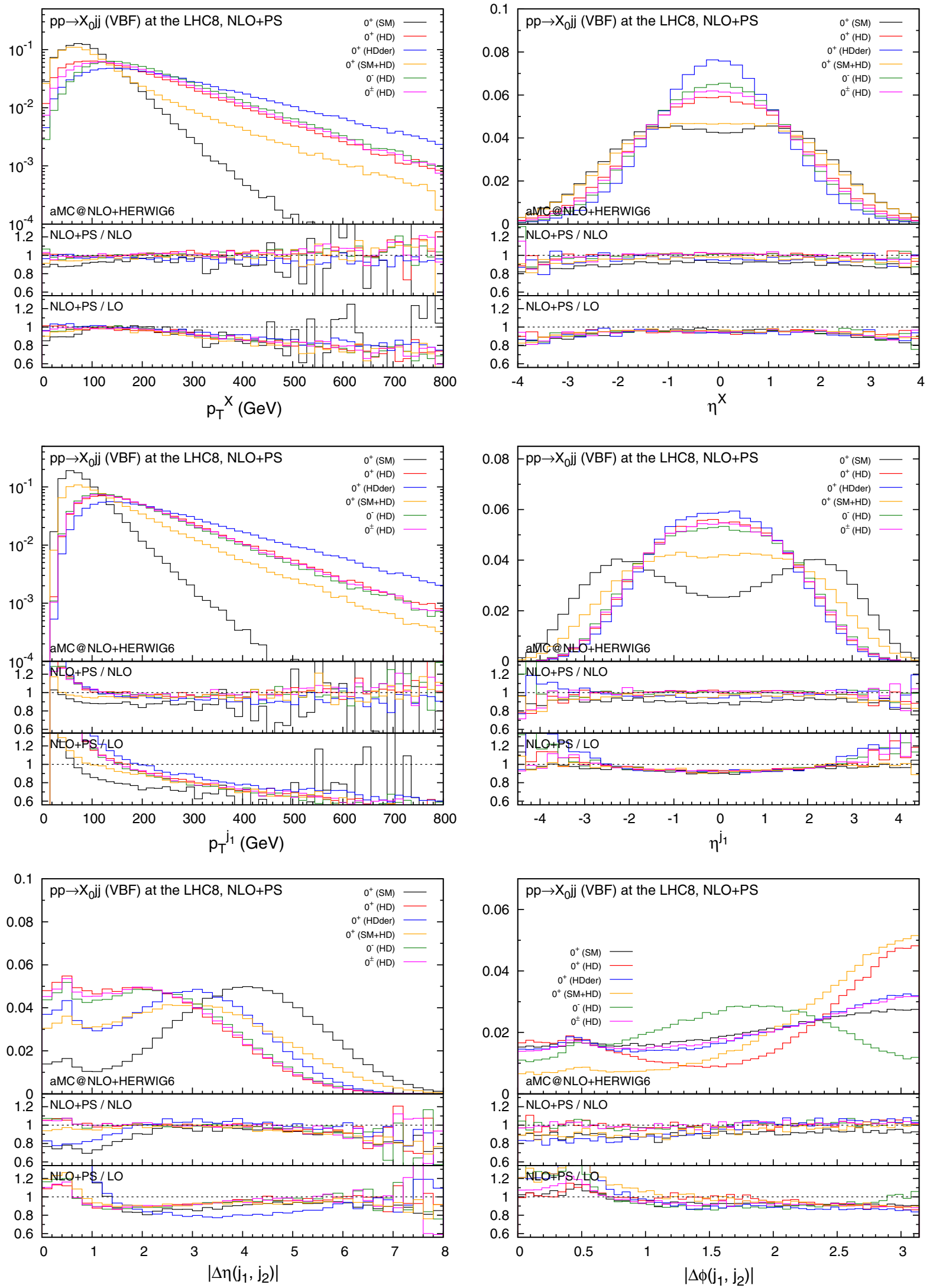

Fig. 2 Distributions for $p_{T}^{X}, \eta^{X}, p_{T}^{j_{1}}, \eta^{j_{1}}, \Delta \eta\left(j_{1}, j_{2}\right)$, and $\Delta \phi\left(j_{1}, j_{2}\right)$ in VBF with the acceptance cuts for the jets. The histograms in the main plots are normalised to unity 

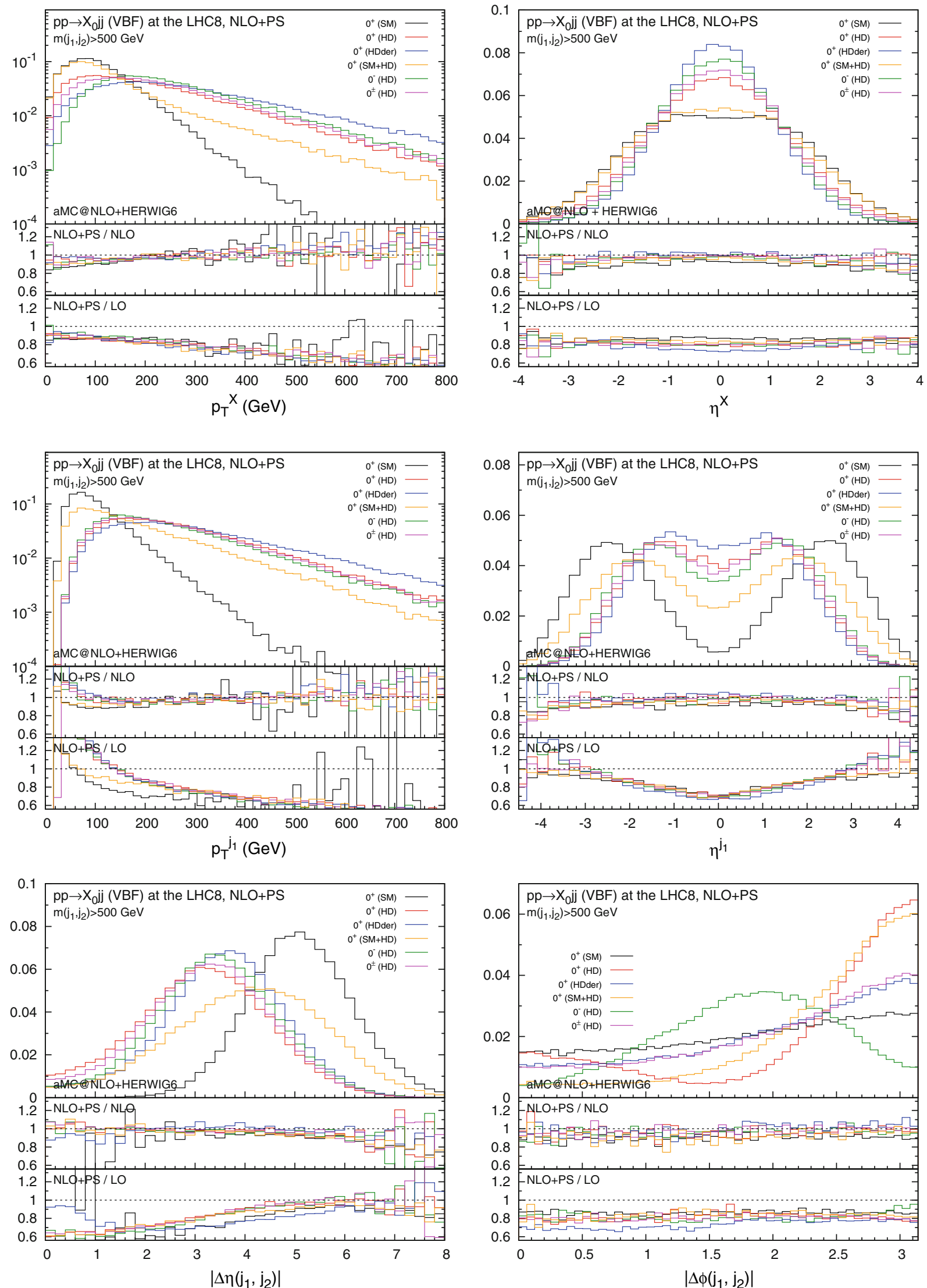

Fig. 3 Same as Fig. 2, but with the additional VBF cut in Eq. (6) 
For the studies on the distributions, we require the presence of at least two reconstructed jets with

$p_{T}^{j}>25 \mathrm{GeV}, \quad\left|\eta^{j}\right|<4.5$.

In addition, we simulate a dedicated VBF selection by imposing an invariant mass cut on the two leading jets,

$m\left(j_{1}, j_{2}\right)>500 \mathrm{GeV}$.

As is well known, such a cut has the scope to minimise the contributions from gluon fusion and allow one to extract VBF couplings. We note that we do not put the rapidity separation cut, although this is the common VBF cut, since $\Delta \eta\left(j_{1}, j_{2}\right)$ itself is a powerful observable to determine the $H V V$ structure in VBF production $[48,85]$.

We start by showing the invariant mass distribution of the two leading jets in Fig. 1 for the six scenarios of Table 3, where the minimal detector cuts in Eq. (5) are applied. With the exception of the scenario featuring the derivative operator (HDder), the distributions are all very similar. This means that the invariant mass cut in Eq. (6), which is imposed in typical VBF selections, acts in a similar way on all scenarios.

The lowest inset in Fig. 1 is the ratio of NLO+PS to LO results, while the middle one shows the ratio of NLO+PS to pure NLO. NLO+PS corrections modify in a consistent way LO parton-level predictions with major effects at high invariant mass, i.e., the QCD corrections tend to make the tagging jets softer. In addition, the parton shower affects both the lower and the higher invariant mass regions.

Figures 2 and 3 collect key plots for the $X_{0}$ and the hardest jet distributions, as well as the rapidity and azimuthal separation of the two leading jets. In Fig. 2 only the acceptance cuts in Eq. (5) are imposed, while in Fig. 3 the additional VBF cut in Eq. (6) is applied. As one can see, the invariant mass cut effectively suppresses the central jet activity, especially for the SM case, while the difference of the distributions among the different scenarios becomes more pronounced.

The unitarity violating behaviour of the higher-dimensional interactions, especially for $0^{+}$(HDder), clearly manifests itself in the transverse momentum distributions for the $X_{0}$ and the jets. The rapidity distribution of the tagging jets displays the fact that in the case of higher-dimensional interactions the jets as a result are much more central than in the SM case. The same glaring difference appears in the azimuthal correlations between the jets which offer clear handle to discriminate about different interactions type and parity assignments.

In all cases NLO corrections are relevant and cannot be described by an overall $K$-factor. Moreover, their impact depends on the applied cuts. Apart from regions in phase space where the jets end up close and therefore are sensitive to NLO/jet reconstruction effects, the parton-shower effect on the shapes is very minor, especially after the VBF cut.
Table $5 p p \rightarrow H\left(W^{+} \rightarrow e^{+} v_{e}\right)$ total cross sections with scale uncertainties and corresponding $K$-factors at $\mathrm{LHC} 8 \mathrm{TeV}$ for various scenarios

\begin{tabular}{llll}
\hline Scenario & $\sigma_{\mathrm{LO}}(\mathrm{fb})$ & $\sigma_{\mathrm{NLO}}(\mathrm{fb})$ & $K$ \\
\hline $0^{+}(\mathrm{SM})$ & $39.58(3)_{-0.6 \%}^{+0.1 \%}$ & $51.22(5)_{-1.8 \%}^{+2.2 \%}$ & 1.29 \\
$0^{+}(\mathrm{HD})$ & $13.51(1)_{-1.7 \%}^{+1.5 \%}$ & $17.51(1)_{-1.3 \%}^{+1.9 \%}$ & 1.30 \\
$0^{+}(\mathrm{HDder})$ & $324.2(2)_{-4.3 \%}^{+4.7 \%}$ & $416.1(4)_{-2.1 \%}^{+2.3 \%}$ & 1.28 \\
$0^{+}(\mathrm{SM}+\mathrm{HD})$ & $118.8(1)_{-2.9 \%}^{+3.0 \%}$ & $154.2(1)_{-1.6 \%}^{+1.8 \%}$ & 1.30 \\
$0^{-}(\mathrm{HD})$ & $8.386(7)_{-2.6 \%}^{+2.6 \%}$ & $10.89(1)_{-1.5 \%}^{+1.8 \%}$ & 1.30 \\
$0^{ \pm}(\mathrm{HD})$ & $10.96(1)_{-2.1 \%}^{+1.9 \%}$ & $14.22(1)_{-1.3 \%}^{+1.8 \%}$ & 1.30 \\
\hline
\end{tabular}

Table 6 Same as Table 5, but for $p p \rightarrow H\left(W^{-} \rightarrow e^{-} \bar{v}_{e}\right)$

\begin{tabular}{llll}
\hline Scenario & $\sigma_{\mathrm{LO}}(\mathrm{fb})$ & $\sigma_{\mathrm{NLO}}(\mathrm{fb})$ & $K$ \\
\hline $0^{+}(\mathrm{SM})$ & $22.46(1)_{-0.6 \%}^{+0.0 \%}$ & $29.86(3)_{-1.8 \%}^{+2.3 \%}$ & 1.33 \\
$0^{+}(\mathrm{HD})$ & $7.009(5)_{-1.7 \%}^{+1.4 \%}$ & $9.355(9)_{-1.3 \%}^{+1.9 \%}$ & 1.34 \\
$0^{+}(\mathrm{HDder})$ & $145.7(1)_{-3.9 \%}^{+4.1 \%}$ & $193.8(1)_{-1.9 \%}^{+2.1 \%}$ & 1.33 \\
$0^{+}(\mathrm{SM}+\mathrm{HD})$ & $57.90(5)_{-2.9 \%}^{+2.8 \%}$ & $77.31(8)_{-1.6 \%}^{+1.8 \%}$ & 1.34 \\
$0^{-}(\mathrm{HD})$ & $4.151(3)_{-2.6 \%}^{+2.5 \%}$ & $5.550(5)_{-1.4 \%}^{+1.7 \%}$ & 1.34 \\
$0^{ \pm}(\mathrm{HD})$ & $5.583(4)_{-2.0}^{+1.8 \%}$ & $7.445(7)_{-1.3 \%}^{+1.8 \%}$ & 1.33 \\
\hline
\end{tabular}

Table 7 Same as Table 5, but for $p p \rightarrow H\left(Z \rightarrow e^{+} e^{-}\right)$

\begin{tabular}{llll}
\hline Scenario & $\sigma_{\mathrm{LO}}(\mathrm{fb})$ & $\sigma_{\mathrm{NLO}}(\mathrm{fb})$ & $K$ \\
\hline $0^{+}(\mathrm{SM})$ & $10.13(1)_{-0.5 \%}^{+0.0 \%}$ & $13.24(1)_{-1.7 \%}^{+2.2 \%}$ & 1.31 \\
$0^{+}(\mathrm{HD})$ & $2.638(2)_{-1.7 \%}^{+1.4 \%}$ & $3.461(3)_{-1.3 \%}^{+1.9 \%}$ & 1.31 \\
$0^{+}(\mathrm{HDder})$ & $48.61(4)_{-3.9 \%}^{+4.2 \%}$ & $63.59(5)_{-1.9 \%}^{+2.1 \%}$ & 1.31 \\
$0^{+}(\mathrm{SM}+\mathrm{HD})$ & $19.95(1)_{-3.1 \%}^{+3.1 \%}$ & $26.24(2)_{-1.6 \%}^{+1.8 \%}$ & 1.32 \\
$0^{-}(\mathrm{HD})$ & $1.480(1)_{-2.7 \%}^{+2.6 \%}$ & $1.952(1)_{-1.5 \%}^{+1.7 \%}$ & 1.32 \\
$0^{ \pm}(\mathrm{HD})$ & $2.061(1)_{-2.0 \%}^{+1.9 \%}$ & $2.705(2)_{-1.3 \%}^{+1.8 \%}$ & 1.31 \\
\hline
\end{tabular}

\section{Vector-boson associated production}

Predictions for Higgs production in association with a weak vector boson in the SM are known up to NNLO accuracy [9092], including EW corrections [93,94]. NLO+PS results can be obtained via (A)MC@NLO $[95,96]$ and the POWHEG BOX [97]. Many phenomenological studies on Higgs spin, parity and couplings are available in the literature $[48,88,98$ 105]. In this section we present the first predictions for EFT interactions including NLO corrections in QCD interfaced with a parton shower in the $\mathrm{VH}$ process.

The code and events for $\mathrm{VH}$ production at hadron colliders can be automatically generated by issuing the following commands:

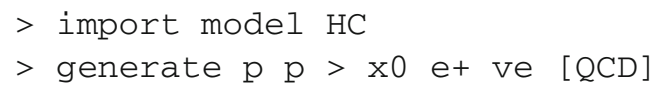



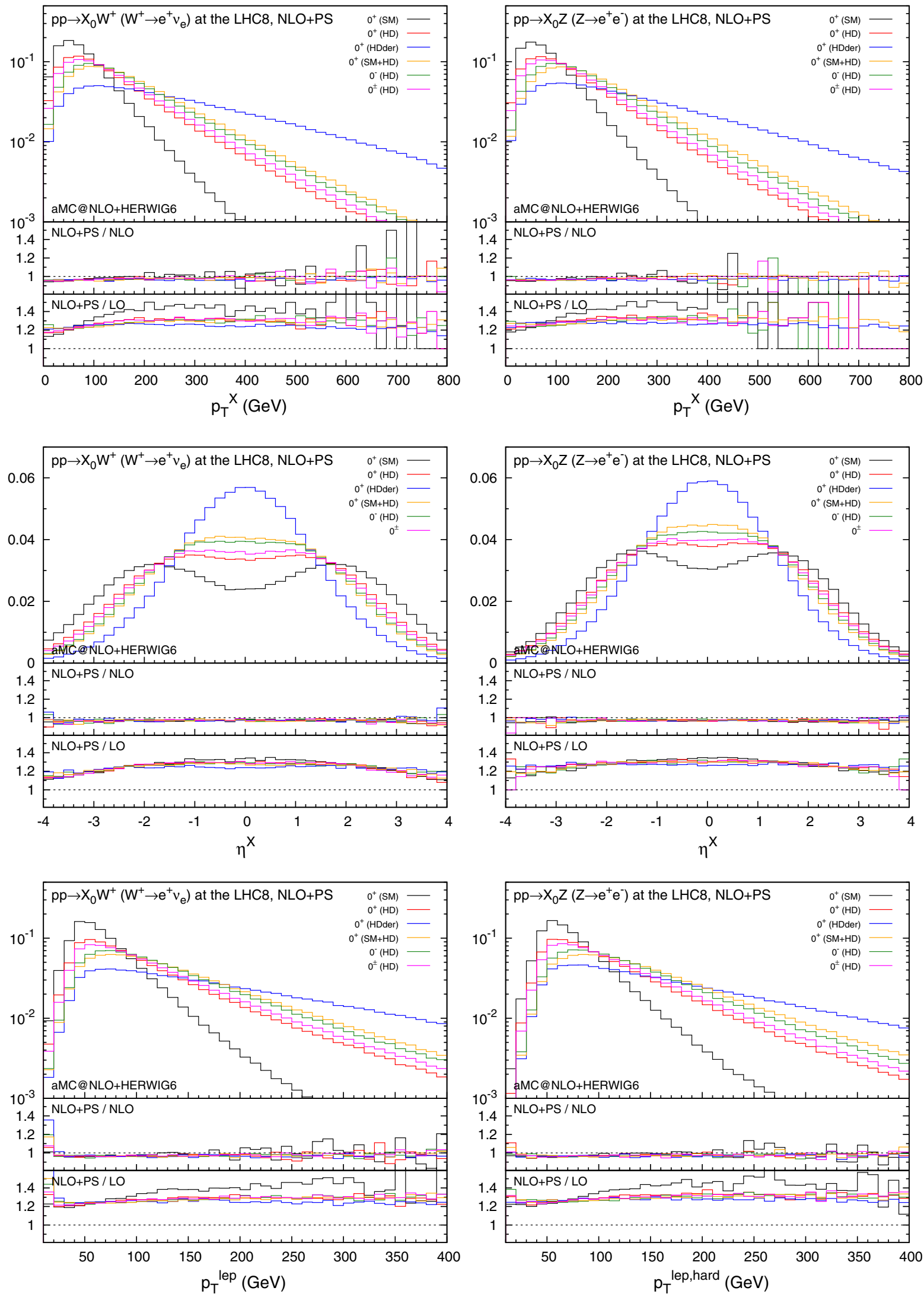

Fig. 4 Distributions for $p_{T}^{X}, \eta^{X}$, and $p_{T}^{\ell}$ in $W^{+} H$ (left) and in $Z H$ (right) production with the acceptance cuts for the lepton(s). The histograms in the main plots are normalised to unity 

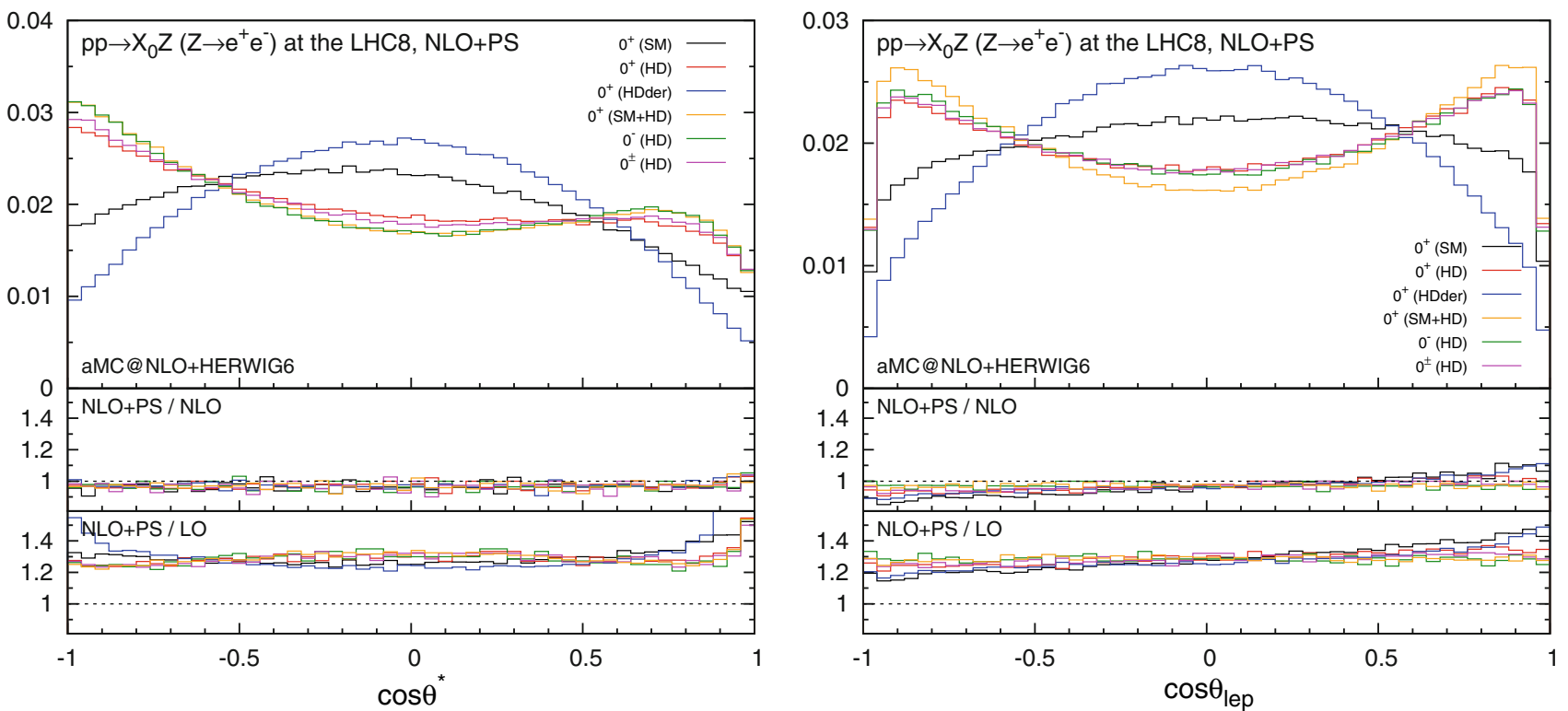

Fig. 5 Distributions for $\cos \theta^{*}$ and $\cos \theta_{\ell}$ in $Z H$ with the acceptance cuts for the leptons. The histograms in the main plots are normalised to unity

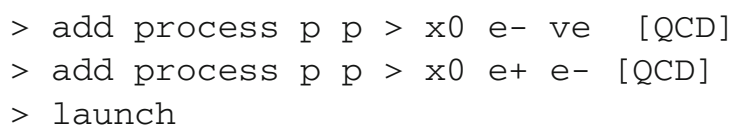

Note that the $W, Z$ decays are performed at the level of the matrix elements and therefore all spin correlations are kept exactly. Again, as in Sect. 3, we do not consider contributions involving the $X_{0} \gamma \gamma$ and $X_{0} Z \gamma$ vertices.

Results for total cross sections (without any cuts) at LO and NLO accuracy and corresponding $K$-factors for the six scenarios defined in Table 3 are collected in Tables 5, 6 and 7 for $p p \rightarrow W^{+} H, W^{-} H$ and $Z H$, respectively, including the $W / Z$ decay branching ratio into a lepton pair. As in the VBF case, the uncertainties correspond to the envelope of independently varying the renormalisation and factorisation scales around the central value $1 / 2<\mu_{R, F} / \mu_{0}<2$ with $\mu_{0}=m_{\mathrm{VH}}$. Apart from the case of the SM for which the uncertainties are accidentally small at LO, the results at NLO display an improved stability. Quite interestingly all $K$ factors are found to be around 1.3 for all the scenarios, with a tiny difference among the processes due to the different initial states. We note that the cancellation of the $s$-channel vector-boson propagator due to the derivative in the higherdimensional scenarios results in the rather large cross section in spite of the $\Lambda=1 \mathrm{TeV}$ cutoff (except for the $0^{+}(\mathrm{SM}+\mathrm{HD}$ ) scenario, where $\Lambda=v=246 \mathrm{GeV}$ ).

We then show, see Fig. 4, the distributions for the several inclusive variables with minimal cuts on the charged lepton(s):

$p_{T}^{\ell}>10 \mathrm{GeV}, \quad\left|\eta^{\ell}\right|<2.5$, for $W^{+} H$ and $Z H$ production (distributions for $W^{-} H$ are very similar to $W^{+} H$ and we do not display them).

The results for $W$ and $Z$ display very similar features. The scenarios that include contributions from higher-dimensional operators show harder $p_{T}$ spectra. This is even more pronounced in the case of the derivative operator (HDder). This fact is also reflected in the shape of the rapidity distributions, i.e., the harder $p_{T}$ spectra correspond to a more central rapidity for the $\mathrm{VH}$ scattering.

As in Sect. 3, the ratios of NLO+PS to LO (NLO) results are presented in the lowest (middle) inset in Fig. 4. NLO+PS effects are quite important when compared with fixed-order LO predictions, and, in many cases, they cannot be accounted for by applying an overall $K$-factor. Conversely, NLO+PS distributions are in almost perfect agreement with fixed-order NLO predictions, witnessing small effects genuinely due to the parton shower.

In Fig. 5 we show the polar angle distributions in $Z H$ production. $\cos \theta^{*}$ is defined as the angle between the intermediate $Z^{*}$ momentum and the reconstructed $Z$ in the $Z^{*}$ rest frame, while $\cos \theta_{\ell}$ is the lepton angle along the $Z$ momentum in the $Z$ rest frame. In this case, NLO+PS corrections do not affect the $\cos \theta^{*}$ distributions significantly, while those of $\cos \theta_{\ell}$ are mildly modified. We note that the asymmetry of the $\cos \theta^{*}$ distribution is due to the cuts on the leptons.

\section{Summary}

We have studied higher-order QCD effects for various spin0 hypotheses in $\mathrm{VBF}$ and $\mathrm{VH}$ production, obtained in a fully automatic way via the model implementation in 
FEYNRULES and event generation at NLO accuracy in the MADGRAPH5_AMC@NLO framework. Our approach to the Higgs characterisation is based on an EFT that takes into account all relevant operators up to dimension six written in terms of fields above the EWSB scale and then expressed in terms of mass eigenstates ( $W, Z, \gamma$ and $H$ ).

We have presented illustrative distributions obtained by interfacing NLO parton-level events to the HERWIG6 parton shower. NLO corrections improve the predictions on the total cross sections by reducing the scale dependence. In addition, our simulations show that NLO+PS effects need to be accounted for to make accurate predictions on the kinematical distributions of the final state objects, such as the Higgs and the jet distributions.

We look forward to the forthcoming LHC experimental studies employing the EFT approach and NLO accurate simulations to extract accurate information on possible new physics effects in Higgs physics.

Acknowledgments We would like to thank all the members of the Higgs Cross Section Working Group for their encouragement in pursuing the Higgs Characterisation project. We also thank Stefano Frixione for helpful comments on the draft. This work has been performed in the framework of the ERC grant 291377 'LHCtheory: Theoretical predictions and analyses of LHC physics: advancing the precision frontier' and it is supported in part by the Belgian Federal Science Policy Office through the Interuniversity Attraction Pole P7/37. The work of FM is supported by the IISN 'MadGraph' convention 4.4511.10, the IISN 'Fundamental interactions' convention 4.4517.08. KM is supported in part by the Strategic Research Program 'High Energy Physics' and the Research Council of the Vrije Universiteit Brussel. The work of MZ is partially supported by the Research Executive Agency (REA) of the European Union under the Grant Agreement number PITN-GA-2010264564 (LHCPhenoNet).

Open Access This article is distributed under the terms of the Creative Commons Attribution License which permits any use, distribution, and reproduction in any medium, provided the original author(s) and the source are credited.

Funded by $\mathrm{SCOAP}^{3}$ / License Version CC BY 4.0.

\section{References}

1. ATLAS Collaboration, G. Aad et al., Observation of a new particle in the search for the Standard Model Higgs boson with the ATLAS detector at the LHC. Phys. Lett. B 716, 1-29 (2012). arXiv:1207. 7214

2. S. Chatrchyan et al., C.M.S. Collaboration, Observation of a new boson at a mass of $125 \mathrm{GeV}$ with the CMS experiment at the LHC. Phys. Lett. B 716, 30-61 (2012). arXiv:1207.7235

3. LHC Higgs Cross Section Working Group Collaboration, S. Dittmaier et al., Handbook of LHC Higgs Cross Sections: 1. Inclusive Observables, arXiv:1101.0593

4. LHC Higgs Cross Section Working Group Collaboration, S. Dittmaier et al., Handbook of LHC Higgs Cross Sections: 2. Differential Distributions, arXiv:1201.3084

5. LHC Higgs Cross Section Working Group Collaboration, S. Heinemeyer et al., Handbook of LHC Higgs Cross Sections: 3. Higgs Properties (2013). doi:10.5170/CERN-2013-004. arXiv: 1307.1347
6. ATLAS Collaboration, Combined coupling measurements of the Higgs-like boson with the ATLAS detector using up to $25 \mathrm{fb}^{-1}$ of proton-proton collision data, ATLAS-CONF-2013-034

7. CMS Collaboration, S. Chatrchyan et al., Observation of a new boson with mass near $125 \mathrm{GeV}$ in pp collisions at $\sqrt{s}=7$ and 8 TeV, JHEP 1306, 081 (2013). arXiv: 1303.4571

8. ATLAS Collaboration, G. Aad et al., Evidence for the spin-0 nature of the Higgs boson using ATLAS data. Phys. Lett. B 726, 120-144 (2013). arXiv:1307.1432

9. CMS Collaboration, S. Chatrchyan et al., Study of the mass and spin-parity of the Higgs Boson candidate via Its decays to Z Boson Pairs. Phys.Rev.Lett. 110, 081803 (2013). arXiv:1212.6639

10. K. Hagiwara, R. Szalapski, D. Zeppenfeld, Anomalous Higgs boson production and decay. Phys. Lett. B 318, 155-162 (1993). arXiv:hep-ph/9308347

11. G. Giudice, C. Grojean, A. Pomarol, R. Rattazzi, The stronglyinteracting light Higgs. JHEP 0706, 045 (2007). arXiv:hep-ph/ 0703164

12. B. Gripaios, A. Pomarol, F. Riva, J. Serra, Beyond the minimal composite Higgs model. JHEP 0904, 070 (2009). arXiv:0902. 1483

13. R. Lafaye, T. Plehn, M. Rauch, D. Zerwas, M. Duhrssen, Measuring the Higgs sector. JHEP 0908, 009 (2009). arXiv:0904.3866

14. I. Low, R. Rattazzi, A. Vichi, Theoretical constraints on the Higgs effective couplings. JHEP 1004, 126 (2010). arXiv:0907.5413

15. D.E. Morrissey, T. Plehn, T.M. Tait, Physics searches at the LHC. Phys. Rep. 515, 1-113 (2012). arXiv:0912.3259

16. R. Contino, C. Grojean, M. Moretti, F. Piccinini, R. Rattazzi, Strong double Higgs production at the LHC. JHEP 1005, 089 (2010). arXiv:1002.1011

17. J. Espinosa, C. Grojean, M. Muhlleitner, Composite Higgs search at the LHC. JHEP 1005, 065 (2010). arXiv:1003.3251

18. A. Azatov, R. Contino, J. Galloway, Model-independent bounds on a light Higgs. JHEP 1204, 127 (2012). arXiv:1202.3415

19. J. Espinosa, C. Grojean, M. Muhlleitner, M. Trott, Fingerprinting Higgs suspects at the LHC. JHEP 1205, 097 (2012). arXiv:1202. 3697

20. J. Ellis, T. You, Global analysis of experimental constraints on a possible Higgs-like particle with mass $125 \mathrm{GeV}$. JHEP 1206, 140 (2012). arXiv:1204.0464

21. M. Klute, R. Lafaye, T. Plehn, M. Rauch, D. Zerwas, Measuring Higgs couplings from LHC data. Phys. Rev. Lett. 109, 101801 (2012). arXiv: 1205.2699

22. I. Low, J. Lykken, G. Shaughnessy, Have we observed the Higgs (imposter)? Phys. Rev. D 86, 093012 (2012). arXiv:1207.1093

23. T. Corbett, O. Eboli, J. Gonzalez-Fraile, M. Gonzalez-Garcia, Constraining anomalous Higgs interactions. Phys. Rev. D 86, 075013 (2012). arXiv:1207.1344

24. J. Ellis, T. You, Global analysis of the Higgs candidate with mass 125 GeV. JHEP 1209, 123 (2012). arXiv:1207.1693

25. M. Montull, F. Riva, Higgs discovery: the beginning or the end of natural EWSB? JHEP 1211, 018 (2012). arXiv:1207.1716

26. J. Espinosa, C. Grojean, M. Muhlleitner, M. Trott, First Glimpses at Higgs' face. JHEP 1212, 045 (2012). arXiv:1207.1717

27. D. Carmi, A. Falkowski, E. Kuflik, T. Volansky, J. Zupan, Higgs after the discovery: a status report. JHEP 1210, 196 (2012). arXiv: 1207.1718

28. T. Plehn, M. Rauch, Higgs couplings after the discovery. Europhys. Lett. 100, 11002 (2012). arXiv: 1207.6108

29. G. Passarino, NLO inspired effective lagrangians for Higgs physics. Nucl. Phys. B 868, 416-458 (2013). arXiv:1209.5538

30. T. Corbett, O. Eboli, J. Gonzalez-Fraile, M. Gonzalez-Garcia, Robust determination of the Higgs couplings: power to the data. Phys. Rev. D 87, 015022 (2013). arXiv: 1211.4580

31. K. Cheung, J.S. Lee, P.-Y. Tseng, Higgs precision (Higgcision) era begins. JHEP 1305, 134 (2013). arXiv:1302.3794 
32. A. Falkowski, F. Riva, A. Urbano, Higgs at last. JHEP 1311, 111 (2013). doi:10.1007/JHEP11(2013)111. arXiv:1303.1812

33. R. Contino, M. Ghezzi, C. Grojean, M. Muhlleitner, M. Spira, Effective Lagrangian for a light Higgs-like scalar. JHEP 1307, 35 (2013). doi:10.1007/JHEP07(2013)035. arXiv:1303.3876

34. Y. Chen, R. Vega-Morales, Extracting effective Higgs couplings in the golden channel. arXiv:1310.2893

35. W. Buchmuller, D. Wyler, Effective lagrangian analysis of new interactions and flavor conservation. Nucl. Phys. B 268, 621 (1986)

36. B. Grzadkowski, M. Iskrzynski, M. Misiak, J. Rosiek, Dimensionsix terms in the standard model lagrangian. JHEP 1010, 085 (2010). arXiv: 1008.4884

37. P. Artoisenet, P. de Aquino, F. Demartin, R. Frederix, S. Frixione, et al., A framework for Higgs characterisation. JHEP 1311, 43 (2013). doi:10.1007/JHEP11(2013)043. arXiv:1306.6464

38. N.D. Christensen, P. de Aquino, C. Degrande, C. Duhr, B. Fuks et al., A Comprehensive approach to new physics simulations. Eur. Phys. J. C 71, 1541 (2011). arXiv:0906.2474

39. N.D. Christensen, C. Duhr, FeynRules-Feynman rules made easy. Comput. Phys. Commun. 180, 1614-1641 (2009). arXiv:0806. 4194

40. A. Alloul, N. D. Christensen, C. Degrande, C. Duhr, B. Fuks, FeynRules 2.0-A complete toolbox for tree-level phenomenology. arXiv: 1310.1921

41. J. Alwall, M. Herquet, F. Maltoni, O. Mattelaer, T. Stelzer, MadGraph 5: going beyond. JHEP 1106, 128 (2011). arXiv:1106.0522

42. R. Frederix, S. Frixione, F. Maltoni, T. Stelzer, Automation of next-to-leading order computations in QCD: the FKS subtraction. JHEP 10, 003 (2009). arXiv:0908.4272

43. V. Hirschi et al., Automation of one-loop QCD corrections. JHEP 05, 044 (2011). arXiv: 1103.0621

44. C. Degrande, C. Duhr, B. Fuks, D. Grellscheid, O. Mattelaer et al., UFO-the universal FeynRules output. Comput. Phys. Commun. 183, 1201-1214 (2012). arXiv: 1108.2040

45. P. de Aquino, W. Link, F. Maltoni, O. Mattelaer, T. Stelzer, Automatic libraries of helicity amplitudes for Feynman diagram computations. Comput. Phys. Commun. 183, 2254-2263 (2012). arXiv: 1108.2041

46. R. Frederix, S. Frixione, V. Hirschi, F. Maltoni, R. Pittau, P. Torrielli, Scalar and pseudoscalar Higgs production in association with a top-antitop pair. Phys. Lett. B 701, 427-433 (2011). arXiv: 1104.5613

47. K. Hagiwara, Q. Li, K. Mawatari, Jet angular correlation in vector-boson fusion processes at hadron colliders. JHEP 0907, 101 (2009). arXiv:0905.4314

48. C. Englert, D. Goncalves-Netto, K. Mawatari, T. Plehn, Higgs quantum numbers in weak Boson fusion. JHEP 1301, 148 (2013). arXiv: 1212.0843

49. A. Alloul, B. Fuks, V. Sanz, Phenomenology of the Higgs effective lagrangian via FeynRules. arXiv: 1310.5150

50. M.L. Mangano, M. Moretti, R. Pittau, Multijet matrix elements and shower evolution in hadronic collisions: $W b \bar{b}+n$ jets as a case study. Nucl. Phys. B 632, 343-362 (2002). arXiv:hep-ph/ 0108069

51. J. Alwall, S. Hoche, F. Krauss, N. Lavesson, L. Lonnblad et al., Comparative study of various algorithms for the merging of parton showers and matrix elements in hadronic collisions. Eur. Phys. J. C 53, 473-500 (2008). arXiv:0706.2569

52. J. Alwall, S. de Visscher, F. Maltoni, QCD radiation in the production of heavy colored particles at the LHC. JHEP 0902, 017 (2009). arXiv:0810.5350

53. S. Frixione, B.R. Webber, Matching NLO QCD computations and parton shower simulations. JHEP 06, 029 (2002). arXiv: hep-ph/0204244
54. S. Frixione, Z. Kunszt, A. Signer, Three jet cross-sections to next-to-leading order. Nucl. Phys. B 467, 399-442 (1996). arXiv: hep-ph/9512328

55. S. Frixione, A general approach to jet cross-sections in QCD. Nucl. Phys. B 507, 295-314 (1997). arXiv:hep-ph/ 9706545

56. G. Ossola, C.G. Papadopoulos, R. Pittau, CutTools: a program implementing the OPP reduction method to compute one-loop amplitudes. JHEP 0803, 042 (2008). arXiv:0711.3596

57. G. Ossola, C.G. Papadopoulos, R. Pittau, Reducing full one-loop amplitudes to scalar integrals at the integrand level. Nucl. Phys. B 763, 147-169 (2007). arXiv:hep-ph/0609007

58. F. Cascioli, P. Maierhofer, S. Pozzorini, Scattering amplitudes with open loops. Phys. Rev. Lett. 108, 111601 (2012). arXiv: 1111.5206

59. A. Martin, W. Stirling, R. Thorne, G. Watt, Parton distributions for the LHC. Eur. Phys. J. C 63, 189-285 (2009). arXiv:0901. 0002

60. M. Cacciari, G.P. Salam, G. Soyez, The Anti-k(t) jet clustering algorithm. JHEP 0804, 063 (2008). arXiv:0802.1189

61. M. Cacciari, G.P. Salam, G. Soyez, FastJet user manual. Eur. Phys. J. C 72, 1896 (2012). arXiv:1111.6097

62. R. Frederix, S. Frixione, V. Hirschi, F. Maltoni, R. Pittau et al., Four-lepton production at hadron colliders: aMC@ NLO predictions with theoretical uncertainties. JHEP 1202, 099 (2012). arXiv: 1110.4738

63. G. Corcella, I. Knowles, G. Marchesini, S. Moretti, K. Odagiri et al., HERWIG 6: an event generator for hadron emission reactions with interfering gluons (including supersymmetric processes). JHEP 0101, 010 (2001). arXiv:hep-ph/0011363

64. M. Bahr, S. Gieseke, M. Gigg, D. Grellscheid, K. Hamilton et al., Herwig++ physics and manual. Eur. Phys. J. C 58, 639-707 (2008). arXiv:0803.0883

65. T. Sjostrand, S. Mrenna, P.Z. Skands, PYTHIA 6.4 physics and manual, JHEP 0605, 026 (2006). arXiv:hep-ph/0603175

66. T. Sjostrand, S. Mrenna, P.Z. Skands, A brief Introduction to PYTHIA 8.1. Comput. Phys. Commun. 178, 852-867 (2008). arXiv:0710.3820

67. S. Frixione, P. Torrielli, M.Zaro, Higgs production through vectorboson fusion at the NLO matched with parton showers. Phys. Lett. B 726, 273-282 (2013). arXiv:1304.7927

68. R.V. Harlander, J. Vollinga, M.M. Weber, Gluon-induced weak Boson fusion. Phys. Rev. D 77, 053010 (2008). arXiv:0801. 3355

69. P. Bolzoni, F. Maltoni, S.-O. Moch, M. Zaro, Higgs production via vector-boson fusion at NNLO in QCD. Phys. Rev. Lett. 105, 011801 (2010). arXiv: 1003.4451

70. P. Bolzoni, F. Maltoni, S.-O. Moch, M. Zaro, Vector boson fusion at NNLO in QCD: SM Higgs and beyond. Phys. Rev. D 85, 035002 (2012). arXiv:1109.3717

71. T. Han, G. Valencia, S. Willenbrock, Structure function approach to vector boson scattering in $\mathrm{p}$ p collisions. Phys. Rev. Lett. 69, 3274-3277 (1992). arXiv:hep-ph/9206246

72. T. Figy, C. Oleari, D. Zeppenfeld, Next-to-leading order jet distributions for Higgs boson production via weak boson fusion. Phys. Rev. D 68, 073005 (2003). arXiv:hep-ph/0306109

73. E.L. Berger, J.M. Campbell, Higgs boson production in weak boson fusion at next-to-leading order. Phys. Rev. D 70, 073011 (2004). arXiv:hep-ph/0403194

74. T. Figy, D. Zeppenfeld, QCD corrections to jet correlations in weak boson fusion. Phys. Lett. B 591, 297-303 (2004). arXiv: hep-ph/0403297

75. V. Hankele, G. Klamke, D. Zeppenfeld, T. Figy, Anomalous Higgs boson couplings in vector boson fusion at the CERN LHC. Phys. Rev. D 74, 095001 (2006). arXiv:hep-ph/0609075 
76. T. Figy, S. Palmer, G. Weiglein, Higgs production via weak Boson fusion in the standard model and the MSSM. JHEP 1202, 105 (2012). arXiv:1012.4789

77. M. Ciccolini, A. Denner, S. Dittmaier, Strong and electroweak corrections to the production of Higgs +2 jets via weak interactions at the LHC. Phys. Rev. Lett. 99, 161803 (2007). arXiv:0707. 0381

78. M. Ciccolini, A. Denner, S. Dittmaier, Electroweak and QCD corrections to Higgs production via vector-boson fusion at the LHC. Phys. Rev. D 77, 013002 (2008). arXiv:0710.4749

79. P. Nason, C. Oleari, NLO Higgs boson production via vectorboson fusion matched with shower in POWHEG. JHEP 1002, 037 (2010). arXiv:0911.5299

80. K. Arnold, M. Bahr, G. Bozzi, F. Campanario, C. Englert et al., VBFNLO: a parton level Monte Carlo for processes with electroweak bosons. Comput. Phys. Commun. 180, 1661-1670 (2009). arXiv:0811.4559

81. K. Arnold, J. Bellm, G. Bozzi, M. Brieg, F. Campanario, et al., VBFNLO: a parton level Monte Carlo for processes with electroweak Bosons-manual for version 2.5.0. arXiv:1107.4038

82. T. Plehn, D.L. Rainwater, D. Zeppenfeld, Determining the structure of Higgs couplings at the LHC. Phys. Rev. Lett. 88, 051801 (2002). arXiv:hep-ph/0105325

83. C. Englert, M. Spannowsky, M. Takeuchi, Measuring Higgs CP and couplings with hadronic event shapes. JHEP 1206, 108 (2012). arXiv: 1203.5788

84. J.R. Andersen, C. Englert, M. Spannowsky, Extracting precise Higgs couplings by using the matrix element method. Phys. Rev. D 87, 015019 (2013). arXiv:1211.3011

85. A. Djouadi, R. Godbole, B. Mellado, K. Mohan, Probing the spinparity of the Higgs boson via jet kinematics in vector boson fusion. Phys. Lett. B 723, 307-313 (2013). arXiv:1301.4965

86. C. Englert, D. Goncalves, G. Nail, M. Spannowsky, The shape of spins. Phys. Rev. D 88, 013016 (2013). doi:10.1103/PhysRevD. 88.013016. arXiv: 1304.0033

87. J. Frank, M. Rauch, D. Zeppenfeld, Higgs spin determination in the WW channel and beyond. arXiv:1305.1883

88. I. Anderson, S. Bolognesi, F. Caola, Y. Gao, A. V. Gritsan, et al., Constraining anomalous HVV interactions at proton and lepton colliders. arXiv:1309.4819

89. P. Artoisenet, R. Frederix, O. Mattelaer, R. Rietkerk, Automatic spin-entangled decays of heavy resonances in Monte Carlo simulations. JHEP 1303, 015 (2013). arXiv: 1212.3460

90. O. Brein, A. Djouadi, R. Harlander, NNLO QCD corrections to the Higgs-strahlung processes at hadron colliders. Phys. Lett. B 579, 149-156 (2004). arXiv:hep-ph/0307206
91. G. Ferrera, M. Grazzini, F. Tramontano, Associated WH production at hadron colliders: a fully exclusive QCD calculation at NNLO. Phys. Rev. Lett. 107, 152003 (2011). arXiv:1107.1164

92. O. Brein, R.V. Harlander, T.J. Zirke, vh@nnlo-Higgs Strahlung at hadron colliders. Comput. Phys. Commun. 184, 998-1003 (2013). arXiv: 1210.5347

93. M. Ciccolini, S. Dittmaier, M. Kramer, Electroweak radiative corrections to associated $\mathrm{WH}$ and $\mathrm{ZH}$ production at Hadron Colliders. Phys. Rev. D 68, 073003 (2003). arXiv:hep-ph/0306234

94. A. Denner, S. Dittmaier, S. Kallweit, A. Muck, Electroweak corrections to Higgs-strahlung off W/Z bosons at the Tevatron and the LHC with HAWK. JHEP 1203, 075 (2012). arXiv:1112.5142

95. S. Frixione, B.R. Webber, The MC@ NLO 3.1 event generator. arXiv:hep-ph/0506182

96. O. Latunde-Dada, MC and NLO for the hadronic decay of Higgs bosons in associated production with vector bosons. JHEP 0905 , 112 (2009). arXiv:0903.4135

97. G. Luisoni, P. Nason, C. Oleari, F. Tramontano, $H W^{ \pm} / \mathrm{HZ}+0$ and 1 jet at NLO with the POWHEG BOX interfaced to GoSam and their merging within MiNLO. JHEP 1310, 083 (2013). arXiv: 1306.2542

98. D. Miller, S. Choi, B. Eberle, M. Muhlleitner, P. Zerwas, Measuring the spin of the Higgs boson. Phys. Lett. B 505, 149-154 (2001). arXiv:hep-ph/0102023

99. N.D. Christensen, T. Han, Y. Li, Testing CP Violation in ZZH Interactions at the LHC. Phys. Lett. B 693, 28-35 (2010). arXiv: 1005.5393

100. N. Desai, D.K. Ghosh, B. Mukhopadhyaya, CP-violating HWW couplings at the Large Hadron Collider. Phys. Rev. D 83, 113004 (2011). arXiv:1104.3327

101. J. Ellis, D.S. Hwang, V. Sanz, T. You, A fast track towards the 'Higgs' spin and parity. JHEP 1211, 134 (2012). arXiv: 1208.6002

102. J. Ellis, V. Sanz, T. You, Associated production evidence against Higgs impostors and anomalous couplings. Eur. Phys. J. C 73, 2507 (2013). arXiv:1303.0208

103. R. Godbole, D.J. Miller, K. Mohan, C.D. White, Boosting Higgs CP properties via VH Production at the Large Hadron Collider. arXiv: 1306.2573

104. G. Isidori, M. Trott, Higgs form factors in associated, production. arXiv: 1307.4051

105. C. Delaunay, G. Perez, H. de Sandes, W. Skiba, Higgs up-down CP asymmetry at the LHC. arXiv: 1308.4930 